\title{
Spectroscopy of four early-type eclipsing binaries ${ }^{\star}$
}

\author{
P. Mayer ${ }^{1}$, R. Lorenz ${ }^{2}$, and H. Drechsel ${ }^{2}$ \\ 1 Astronomical Institute of the Charles University, V Holešovičkách 2, 18000 Praha 8, Czech Republic \\ 2 Dr. Remeis-Sternwarte Bamberg, Astronomisches Institut der Universität Erlangen-Nürnberg, \\ Sternwartstraße 7, 96049 Bamberg, Germany \\ e-mail: lorenz/drechsel@sternwarte.uni-erlangen.de
}

Received 25 February 2002 / Accepted 25 March 2002

\begin{abstract}
New spectroscopic data were obtained for the early-type binaries V337 Aql, V649 Cas, V382 Cyg, and V431 Pup. Their radial velocity curves are presented. These are the first such data for V337 Aql and V431 Pup. In the case of V382 Cyg, our data confirm previously obtained results. Especially for V649 Cas our radial velocity curve yields important new implications for the component masses: the detection of third body lines, which are blended with the lines of the primary component, solves the problem of the unacceptably small masses formerly deduced for this binary; now we determine masses closer to the values expected for spectral type B 0. V431 Pup is an important object, because it is a new member of the small group of early-type eclipsing binaries with an evolved component. A published light curve of V337 Aql is solved by the MORO code, and system parameters are derived. The period of V649 Cas is found to be variable, and a more accurate period is calculated for V431 Pup.
\end{abstract}

Key words. binaries: eclipsing - binaries: spectroscopic - stars: early-type - stars: fundamental parameters stars: individual: V337 Aql, V649 Cas, V382 Cyg, V431 Pup

\section{Introduction}

In this paper, new spectroscopic results for V337 Aql, V649 Cas, V382 Cyg, and V431 Pup are presented. Though various spectroscopic investigations of these eclipsing binary systems can be found in literature, there were reasons to revisit these objects on the basis of new observations: for V337 Aql and V431 Pup no orbits had been published yet; for V649 Cas the presence of a third body was already suggested by van Hamme (1992) if confirmed, the strange masses found for this binary would eventually change to acceptable values; in case of V382 Cyg, rather different values of the radial velocity semi-amplitudes appeared in studies published by now.

The composite spectral type of our stars was classified as B 0.5p for V337 Aql (Hiltner 1956), B 0 Vn for V649 Cas (Cowley 1972), O 8 for V382 Cyg (Hiltner 1956), and B 1 III for V431 Pup (Feast et al. 1955). A rather wide range of deviating masses, partly inconsistent with the spectral types, had been given for such stars in previous

Send offprint requests to: P. Mayer,

e-mail: mayer@mbox.cesnet.cz

* Based on observations collected at the European Southern Observatory, La Silla, Chile, and at the German-Spanish Astronomical Center (DSAZ), Calar Alto, operated by the Max-Planck-Institut für Astronomie Heidelberg jointly with the Spanish National Commission for Astronomy. studies. New investigations appeared necessary to clarify such discrepancies.

\section{Spectroscopic data}

For the stars V337 Aql, V649 Cas, and V382 Cyg, spectra were obtained at the Calar Alto Observatory (Spain) with the coudé spectrograph of the $2.2 \mathrm{~m}$ telescope, yielding a reciprocal dispersion of $8.5 \AA \mathrm{mm}^{-1}$ and a resolving power of 24000 to 32000 (for further details see Lorenz et al. 1998). In most cases, spectra were taken around quadrature phases, since the orbits could be assumed as circular.

For the star V431 Pup we obtained spectra at the ESO La Silla Observatory (Chile). The ESO $1.52 \mathrm{~m}$ telescope with ECHELEC was used in the years 1992 and 1993, with a reciprocal dispersion of $3.8 \AA \mathrm{mm}^{-1}$, and a resolving power of 23000 to 32000 . In 1994, the CAT/CES equipment was used, with a reciprocal dispersion of $2.6 \AA \mathrm{mm}^{-1}$ and a resolving power of about 56000 (for further details see Mayer et al. 1997).

For a journal of the spectra of our four program stars together with measured radial velocities see Table 1. Radial velocities of the binary components were derived by fitting multiple Gaussian profiles to blended features. In cases where the lines of both components were clearly separated, also the SPEFO code was used (this code 


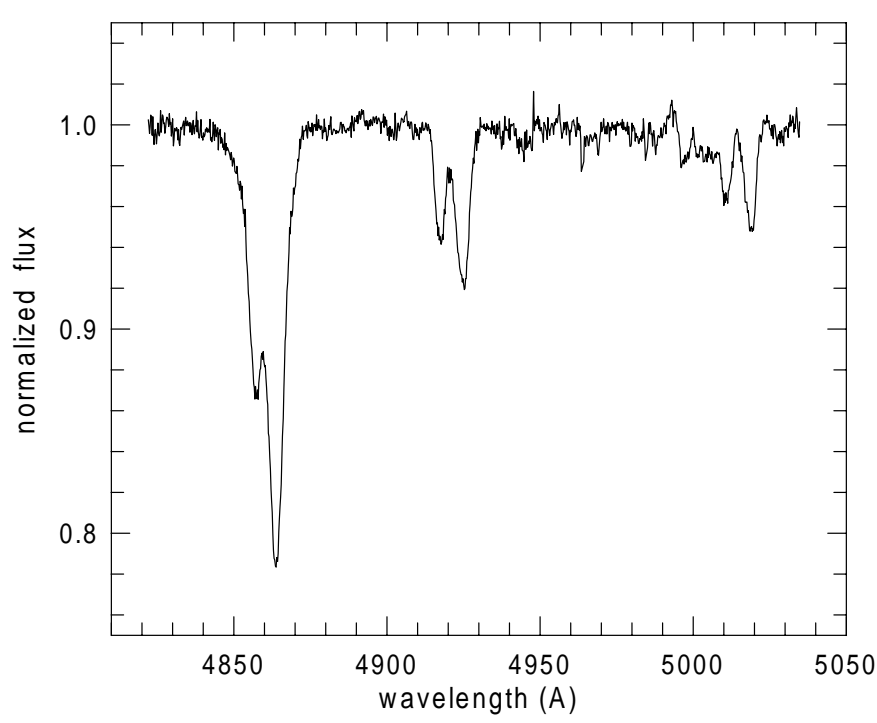

Fig. 1. Spectrum of V337 Aql at phase 0.766.

compares the line profile with its reflection, see Horn et al. 1996 and Škoda 1996).

\section{V337 Aql}

V337 Aql (HD 177284) has been known as an eclipsing variable for a long time. A photoelectric light curve at an effective wavelength of $\lambda=5150 \AA$ was obtained by Catalano et al. (1971). Later, Alduseva (1977) presented $U B V$ light curves, which, however, suffer from a rather poor phase coverage. The period of the binary was studied, among others, by Mayer et al. (1998). The period is not constant, and reasons for its variability so far remain unclear. One conceivable explanation is the suggested lighttime effect due to a third body. For the phasing of our radial velocities the following linear ephemeris was used:

Prim. min. $=$ HJD $2448779.5175+2 \mathrm{~d} 7338794 \cdot E$.

Radial velocities were already measured by Neubauer (1943) and Feast \& Thackeray (1963). However, in the first paper only the mean of 6 measurements was given, and no variability of the radial velocity was recognized. Feast \& Thackeray first classified the spectra as being of SB2 type and measured radial velocities on plates from 4 nights (but the lines appeared resolved into two components on one plate only).

An example of a spectrum is given in Fig. 1. Only $\mathrm{H} \beta$ and He I (4922 and 5015) lines are present. When measuring radial velocities, we noticed that the $\mathrm{H} \beta$ line profile strongly deviated from a simple Gaussian, while an approximation of the observed profile by two Gaussians of different widths and depths gave a reasonably good representation of the line features. Velocities of the primary component of the $\mathrm{H} \beta$ line are systematically more negative by about $16 \mathrm{~km} \mathrm{~s}^{-1}$ compared to the same component of the He I 4922 line. Most probably, this effect is due to the contribution of the Pickering He II $4859.32 \AA$

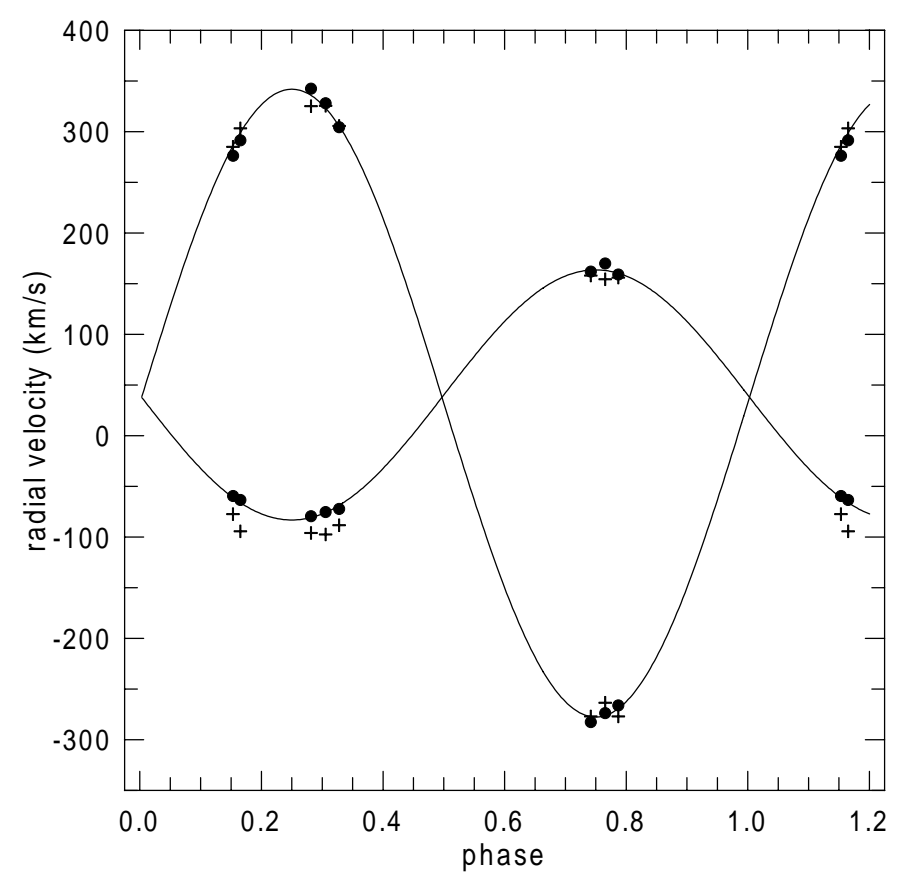

Fig. 2. Radial velocity curve for V337 Aql; filled circles - He I; crosses $-\mathrm{H} \beta$.

line. The secondary component is not well separated, and hence its velocities less certain. The same behaviour was observed by us in the case of the O 8-type binary AB Cru (Lorenz et al. 1994), where this systematic deviation reached $27.7 \mathrm{~km} \mathrm{~s}^{-1}$. Unfortunately, we do not know the strength of other He II lines to study the effect of blending of hydrogen Balmer lines with He II components on the radial velocities more quantitatively.

The He I line components are well separated, and for the 4922 line easily measurable. The mean difference of both methods mentioned above (SPEFO versus GAUSS) is $+0.9 \mathrm{~km} \mathrm{~s}^{-1}$ for the primary and $+1.7 \mathrm{~km} \mathrm{~s}^{-1}$ for the secondary. Averages from both methods are given in Table 1. However, the primary component in the 5015 line always exhibits some asymmetry.

Due to these reasons, we consider only the velocities measured for the 4922 line as reliable. The corresponding radial velocity curve is shown in Fig. 2 and the resulting parameters of the orbit are given in Table 2. The value of inclination $i=86^{\circ}$ found by Catalano et al. (1971) appeared us rather uncertain, so we solved the light curve published by Catalano et al. again using the MORO code (Drechsel et al. 1995). The results are given in Table 3. The normal points as given by Catalano et al. at an effective wavelength of $5150 \AA$ together with the best fit solution (solid line) are shown in Fig. 3. The corresponding system configuration in terms of a meridional intersection of Roche equipotentials is displayed in Fig. 4. The system is semi-detached, with the secondary filling its critical Roche volume. 
Table 1. Journal of new spectra and measured radial velocities.

\begin{tabular}{|c|c|c|c|c|c|c|c|c|c|}
\hline $\begin{array}{l}\text { JD (mid-exp.) } \\
-2400000\end{array}$ & $\begin{array}{l}\text { exp. time } \\
(\min )\end{array}$ & phase $^{a}$ & $\begin{array}{l}\text { spectral } \\
\text { region }\end{array}$ & & & & & & \\
\hline V337 Aql: & & & & Prim.: 4922 & Sec.: 4922 & Prim: $\mathrm{H} \beta$ & Sec.: $\mathrm{H} \beta$ & & \\
\hline 49909.374 & 90 & 0.2816 & $4826-5035$ & -79.6 & 342.6 & -96.0 & 325.3 & & \\
\hline 49909.439 & 90 & 0.3054 & $4826-5035$ & -75.3 & 328.3 & -97.4 & 325.7 & & \\
\hline 49909.500 & 60 & 0.3277 & $4826-5035$ & -72.2 & 304.5 & -88.3 & 305.8 & & \\
\hline 49913.366 & 90 & 0.7418 & $4826-5035$ & 161.9 & -282.6 & 158.1 & -277.1 & & \\
\hline 49913.431 & 90 & 0.7656 & $4826-5035$ & 170.0 & -273.8 & 154.3 & -263.4 & & \\
\hline 49913.490 & 60 & 0.7871 & $4826-5035$ & 159.1 & -266.2 & 156.0 & -276.9 & & \\
\hline 49914.491 & 60 & 0.1533 & $4826-5035$ & -59.4 & 276.4 & -77.4 & 285.2 & & \\
\hline 49914.524 & 30 & 0.1654 & $4826-5035$ & -63.5 & 291.5 & -94.4 & 303.4 & & \\
\hline V649 Cas: & & & & Prim.: 4922 & Sec.: 4922 & & & & \\
\hline 49907.434 & 30 & 0.3078 & $6510-6720$ & -117.1 & 244.2 & & & & \\
\hline 49907.559 & 20 & 0.3601 & $4668-4880$ & -110.1 & & & & & \\
\hline 49907.633 & 30 & 0.4036 & $4826-5035$ & -77.9 & 159.7 & & & & \\
\hline 49908.397 & 20 & 0.7105 & $4826-5035$ & 103.7 & -285.0 & & & & \\
\hline 49908.424 & 40 & 0.7218 & $4826-5035$ & 104.9 & -287.9 & & & & \\
\hline 49908.451 & 30 & 0.7331 & $4826-5035$ & 106.2 & -287.9 & & & & \\
\hline 49908.481 & 40 & 0.7456 & $4826-5035$ & 104.9 & -284.9 & & & & \\
\hline 49908.560 & 40 & 0.7787 & $4826-5035$ & 108.0 & -291.0 & & & & \\
\hline 49908.590 & 40 & 0.7912 & $4826-5035$ & 104.9 & -285.0 & & & & \\
\hline 49909.631 & 15 & 0.2266 & $4826-5035$ & -125.4 & 263.2 & & & & \\
\hline 49909.642 & 15 & 0.2312 & $4826-5035$ & -126.6 & 260.2 & & & & \\
\hline 49914.435 & 30 & 0.2355 & $4826-5035$ & -126.2 & 257.5 & & & & \\
\hline 49914.456 & 25 & 0.2443 & $4826-5035$ & -126.2 & 251.4 & & & & \\
\hline 49915.675 & 20 & 0.7541 & $4826-5035$ & 109.3 & -290.9 & & & & \\
\hline V382 Cyg: & & & & Prim.: 4542 & Sec: 4542 & Prim.: 4686 & Sec.: 4686 & Prim.: $\mathrm{H} \beta$ & Sec.: $\mathrm{H} \beta$ \\
\hline 49526.378 & 60 & 0.7020 & $4524-4736$ & 257.4 & -349.7 & 277.0 & -362.6 & & \\
\hline 49526.433 & 60 & 0.7312 & $4524-4736$ & 271.1 & -339.6 & 275.6 & -362.0 & & \\
\hline 49526.475 & 60 & 0.7535 & $4524-4736$ & 268.1 & -344.5 & 283.0 & -356.6 & & \\
\hline 49526.525 & 60 & 0.7800 & $4524-4736$ & 273.0 & -338.0 & 277.1 & -336.9 & & \\
\hline 49526.643 & 60 & 0.8426 & $4666-4879$ & & & 233.5 & -282.9 & 203.3 & -296.6 \\
\hline 49527.365 & 60 & 0.2255 & $4524-4736$ & -241.6 & 374.7 & -257.2 & 370.6 & & \\
\hline 49527.412 & 60 & 0.2504 & $4524-4736$ & -241.7 & 383.2 & -259.1 & 376.3 & & \\
\hline 49527.458 & 60 & 0.2748 & $4524-4736$ & -231.5 & 378.0 & -260.1 & 383.3 & & \\
\hline 49527.496 & 45 & 0.2950 & $4524-4736$ & -221.3 & 373.9 & -247.1 & 376.5 & & \\
\hline 49528.363 & 60 & 0.7548 & $4674-4888$ & & & 261.0 & -349.5 & 233.8 & -362.6 \\
\hline 49528.409 & 60 & 0.7792 & $4674-4888$ & & & 254.1 & -333.6 & 223.8 & -358.7 \\
\hline $49529.362^{b}$ & 60 & 0.2846 & $6515-6725$ & & & & & -254.0 & 308.8 \\
\hline 49530.644 & 30 & 0.9645 & $6515-6725$ & & & & & & \\
\hline 49908.365 & 60 & 0.2907 & $4826-5035$ & & & & & -263.4 & 316.3 \\
\hline 49915.644 & 60 & 0.1512 & $4826-5035$ & & & & & -241.1 & 270.9 \\
\hline V431 Pup: & & & & Prim.: 4713 & Prim.: 4922 & Sec.: 4922 & Prim.: $\mathrm{H} \beta$ & Prim.: $\mathrm{H} \alpha$ & \\
\hline 48674.696 & 65 & 0.7436 & $4590-4905$ & -73.7 & & & & & \\
\hline 48678.613 & 65 & 0.1618 & $4590-4905$ & 140.6 & & & & & \\
\hline 48679.615 & 65 & 0.2688 & $4590-4905$ & 127.6 & & & & & \\
\hline 49024.538 & 60 & 0.0969 & $4628-4953$ & 138.2 & 149.9 & -206 & & & \\
\hline 49026.595 & 50 & 0.3165 & $4628-4953$ & 87.4 & 96.3 & & & & \\
\hline 49029.610 & 60 & 0.6385 & $4628-4953$ & -49.5 & -52.1 & 199 & & & \\
\hline 49146.470 & 60: & 0.1154 & $4826-5143$ & & 153.0 & -162 & 140.0 & & \\
\hline 49148.494 & 60: & 0.3315 & $4826-5143$ & & & & 69.3 & & \\
\hline 49151.461 & 60: & 0.6483 & $4826-5143$ & & -70.8 & & -77.8 & & \\
\hline 49449.534 & 30 & 0.4746 & $4903-4942$ & & 21.9 & & & & \\
\hline 49450.565 & 30 & 0.5846 & $4903-4942$ & & -32.5 & & & & \\
\hline 49451.518 & 30 & 0.6863 & $4903-4942$ & & -68.9 & 144 & & & \\
\hline 49452.559 & 45 & 0.7974 & $6534-6592$ & & & & & -67.3 & \\
\hline
\end{tabular}

${ }^{a}$ Heliocentric correction applied.

${ }^{b}$ Instead of $\mathrm{H} \beta$, radial velocities measured for $\mathrm{H} \alpha$ are given. 
Table 2. Parameters of V337 Aql, V649 Cas and V382 Cyg.

\begin{tabular}{cccccccc}
\hline \hline \multirow{2}{*}{ Parameter } & \multirow{2}{*}{ unit } & \multicolumn{2}{c}{ V337 Aql } & \multicolumn{2}{c}{ V649 Cas } & \multicolumn{2}{c}{ V382 Cyg } \\
& & individual $V \gamma$ & common $V \gamma$ & individual $V \gamma$ & common $V \gamma$ & individual $V \gamma$ & common $V \gamma$ \\
\hline$K_{1}$ & $\mathrm{~km} \mathrm{~s}^{-1}$ & 123.4 & 122.8 & 116.8 & 117.4 & 267 & 268 \\
$K_{2}$ & $\mathrm{~km} \mathrm{~s}^{-1}$ & 309.8 & 308.9 & 275.8 & 276.5 & 367 & 367 \\
$V_{1} \gamma$ & $\mathrm{km} \mathrm{s}^{-1}$ & +40.2 & 37.5 & -9.0 & -11.7 & +7.8 & +9.9 \\
$V_{2} \gamma$ & $\mathrm{km} \mathrm{s}^{-1}$ & +32.2 & 37.5 & -15.8 & -11.7 & +14.3 & +9.9 \\
$a \sin i$ & $R_{\odot}$ & & 23.4 & & 18.6 & & 23.6 \\
$m_{1} \sin ^{3} i$ & $M_{\odot}$ & & 16.52 & & 10.6 & 29.0 \\
$m_{2} \sin ^{3} i$ & $M_{\odot}$ & & 6.58 & & 4.5 & 21.0 \\
\hline
\end{tabular}

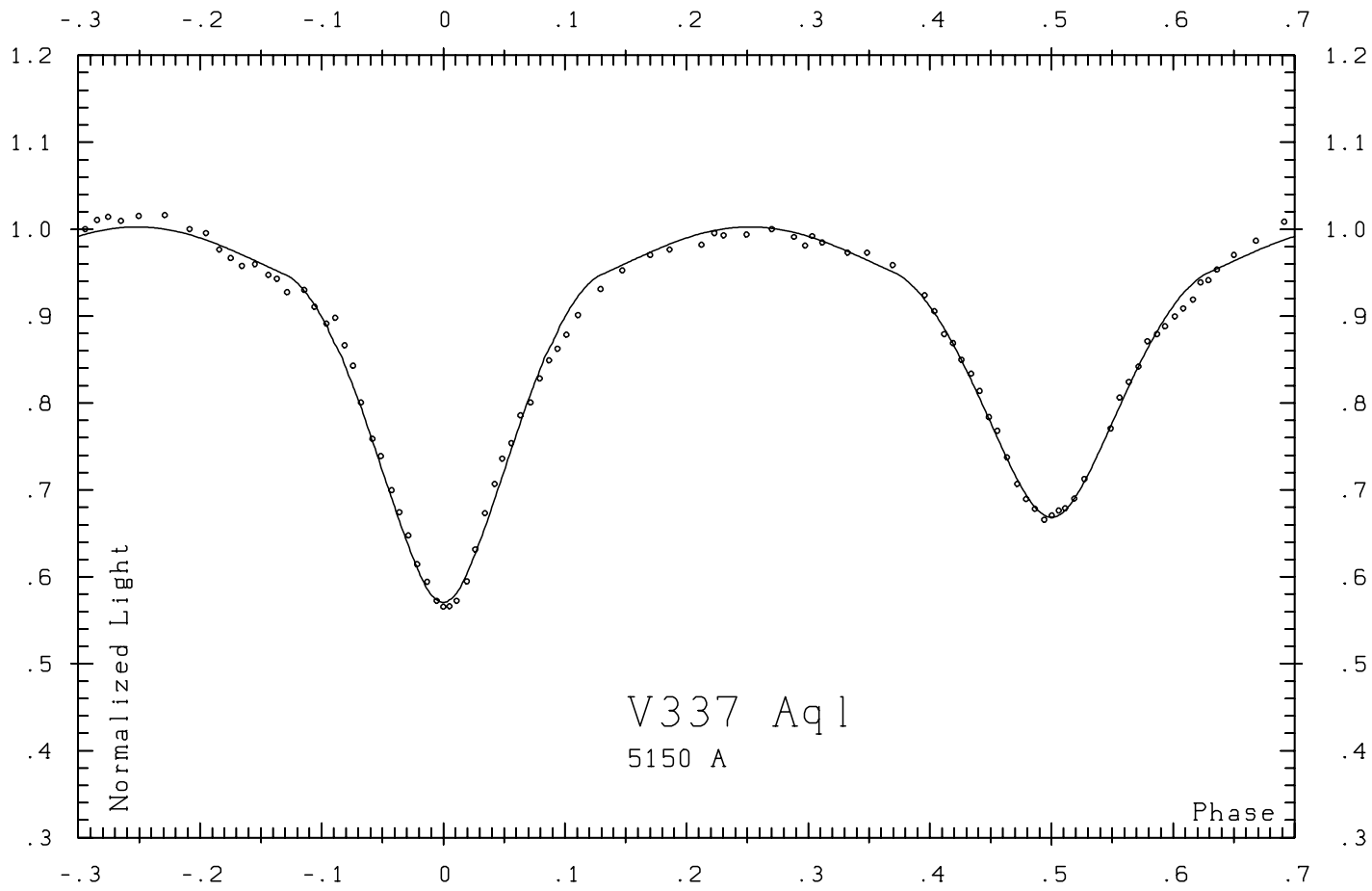

Fig. 3. Light curve of V337 Aql obtained by Catalano et al. (1971) at an effective wavelength of $5150 \AA$ (open circles: normal points) together with the best fit MORO solution (solid line).

\section{V337 Aql}
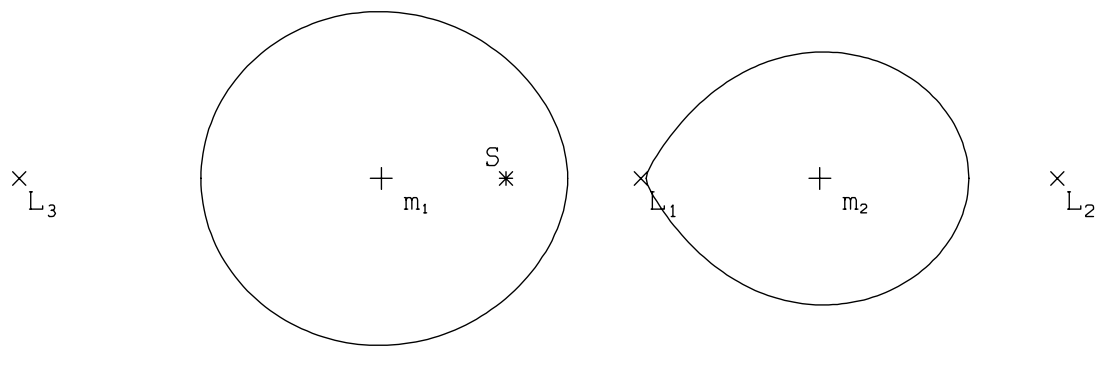

$$
\begin{array}{lll}
\mathrm{q}=0.3980 & \Omega_{1}=2.9392 & \Omega\left(L_{1}\right)=2.6228 \\
\delta_{1}=0.0229 & \Omega_{2}=2.6244 & \Omega\left(L_{2}\right)=2.4217 \\
\delta_{2}=0.0129 & & \Omega\left(L_{3}\right)=2.2066
\end{array}
$$

Fig. 4. Semi-detached system configuration of V337 Aql corresponding to the light curve solution shown in Fig. 3; solution parameters are given in Table 3. 
Table 3. Light curve solution of V337 Aql.

\begin{tabular}{ll}
\hline \hline \multicolumn{2}{l}{ Fixed parameters: } \\
\hline$q\left(=M_{2} / M_{1}\right)$ & 0.398 \\
$T_{\text {eff }}(1)$ & $28000 \mathrm{~K}$ \\
$A_{1}{ }^{a}$ & 1.0 \\
$A_{2}{ }^{a}$ & 1.0 \\
$g_{1}{ }^{b}$ & 1.0 \\
$g_{2}{ }^{b}$ & 1.0 \\
$x_{1}(\lambda 5150)^{c}$ & 0.26 \\
$x_{2}(\lambda 5150)^{c}$ & 0.29 \\
\hline Adjusted parameters: $^{2}$ & $80.7 \pm 0{ }^{\circ} 5$ \\
\hline$i$ & $23900 \mathrm{~K} \pm 700 \mathrm{~K}$ \\
$T_{\text {eff }}(2)$ & $2.939 \pm 0.057$ \\
$\Omega_{1}$ & $2.624 \pm 0.024$ \\
$\Omega_{2}$ & $0.696 \pm 0.013$ \\
$L_{1}(\lambda 5150)^{d}$ & 0.000 \\
$l_{3}(\lambda 5150)$ & $0.023 \pm 0.013$ \\
$\delta_{1}{ }^{e}$ & $0.013 \pm 0.005$ \\
$\delta_{2}{ }^{f}$ &
\end{tabular}

\begin{tabular}{ll}
\hline Roche radii: ${ }^{g}$ & \\
\hline$r_{1}$ (pole) & $0.381 \pm 0.008$ \\
$r_{1}$ (point) & $0.425 \pm 0.014$ \\
$r_{1}$ (side) & $0.397 \pm 0.023$ \\
$r_{1}$ (back) & $0.411 \pm 0.011$ \\
$r_{2}$ (pole) & $0.288 \pm 0.005$ \\
$r_{2}$ (point) & $0.391 \pm 0.005$ \\
$r_{2}$ (side) & $0.302 \pm 0.006$ \\
$r_{2}$ (back) & $0.394 \pm 0.009$ \\
\hline
\end{tabular}

a Bolometric albedo.

${ }^{b}$ Gravitational darkening exponent.

${ }^{c}$ Linear limb darkening coefficient; theoretical value taken from Wade \& Rucinski (1985).

${ }^{d}$ Relative luminosity $L_{1} /\left(L_{1}+L_{2}\right)$.

$e$ Fraction of third light at maximum.

$f$ Radiation pressure parameter, see Drechsel et al. (1995).

$g$ Fractional Roche radius (relative to separation of mass centers).

\section{V649 Cas}

V649 Cas (HR 8854; HD 219634) has been known as a spectroscopic binary since the work of Young (1939). It was discovered as an eclipsing binary by Jerzykiewicz \& Sterken (1982) and independently by Gulliver et al. (1982). Radial velocities were published by Gulliver et al. (1985). $B V$ light curves were obtained and solved by Martin et al. (1990). A solution of the light curve was also published by van Hamme (1992), who suggested that quite a large amount of third light - 40\% - should be present in this system. Also the resulting masses were too low for the given spectral type. Note that there are several cases where originally erroneous masses were deduced due to the presence of third body lines: e.g., LY Aur (Popper 1982), SZ Cam (Mayer et al. 1994; Lorenz et al. 1998; Harries et al. 1997) and V1182 Aql (Lorenz et al. 1997, and a

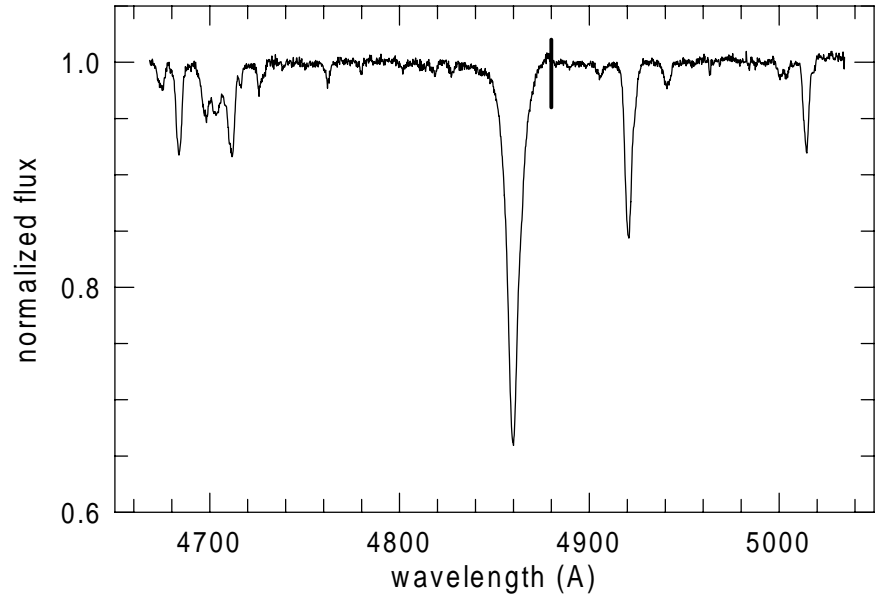

Fig. 5. Spectrum of V649 Cas at phase 0.360 (4670 to $4880 \AA$ ) and 0.404 (4880 to $5036 \AA$ ); the exposures are separated by a vertical bar.

Table 4. Times of minima of V649 Cas.

\begin{tabular}{lcrrc}
\hline \hline HJD-2400000 & m.e. (d) & Epoch & O-C (d) & Source \\
\hline 47099.3861 & & 0 & +0.0209 & 1 \\
48500.6277 & & 586 & 0.0000 & 2 \\
52171.170 & 0.005 & 2121 & 0.0000 & 3 \\
\hline${ }^{1}$ Martin et al. (1990). & & & \\
${ }^{2}$ HIPPARCOS. & & & \\
${ }^{3}$ Hvar, this paper. & & &
\end{tabular}

paper in preparation). Measurements by Jerzykiewicz were published in graphical form (Jerzykiewicz 1993); only a few measurements were taken during primary eclipse, so that a light curve solution and determination of the period are hardly possible.

There are some doubts concerning the period of this binary. Gulliver et al. (1985) give 2. $391253 \pm 0$ d.000002. This value is based on a series of radial velocity measurements covering 3300 days, so its actual accuracy is about one order of magnitude worse. Using the $B V$ data published by Martin et al. (1990), van Hamme (1992) found "a phase shift of 0.9988 "; we got a similar value. Choosing an epoch near the middle of the time interval covered by the Martin et al. measurements, the zero epoch time given in Table 4 can be calculated. According to the HIPPARCOS catalogue, another time of minimum is HJD 2448500.5980. With the van Hamme ephemeris, such a value gives a rather large $\mathrm{O}-\mathrm{C}=-0 \mathrm{~d} 0433$. However, if the Kweevan Woerden method (Kwee \& van Woerden 1956) is applied to the HIPPARCOS photometric measurements, a somewhat different time results (Table 4).

On request by the present authors the star was observed at Hvar Observatory during the second half of 2001. From several nights a normal minimum was calculated. For the phasing of our spectroscopic measurements, the second and third minimum times as given in Table 4 were 


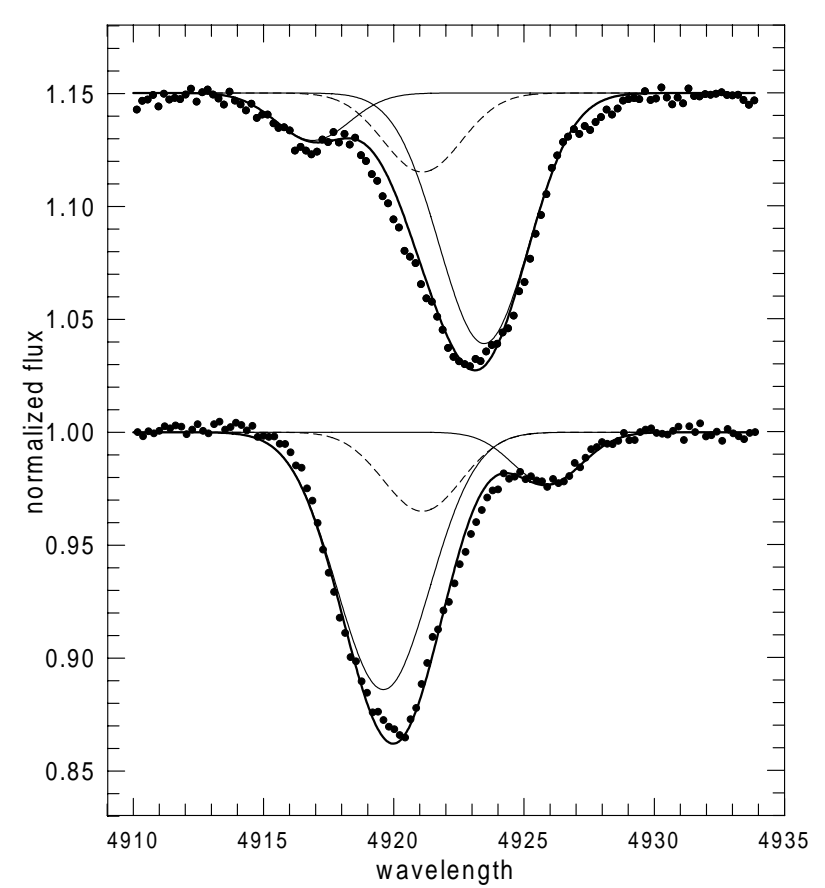

Fig. 6. Profiles of the He I 4922 line in V649 Cas at phases 0.244 and 0.754 (shifted in flux by +0.15 ). The Gaussian fits for the three components are shown. Points represent the observed spectrum, dashed line the third component, thick line the resulting profile.

used to yield an ephemeris valid during the time of our spectroscopic observations:

Prim. Min. $=$ HJD $2448500.6277+2.391233 \cdot E$.

Due to line blending effects the time of conjunction calculated from old radial velocities appears unreliable. The rather large $\mathrm{O}-\mathrm{C}$ in the first line of Table 4 indicates that the period of V649 Cas is probably variable.

An example of a V649 Cas spectrum is presented in Fig. 5. Profiles of the line He I 4922 in spectra taken near quadratures (see Fig. 6) actually show the expected asymmetry of the primary line, which is conceivably caused by the third component. The lines were fitted with Gaussians. The third line position had to be kept fixed, and a position at $\lambda=4921.1 \AA$ appeared as an acceptable compromise between positions suggested by spectra taken at opposite quadratures $^{1}$. The $F W H M$ of the line was assumed as $2.4 \AA$ and its depth as 0.08 of the continuum. Note that a similar process had to be applied also in the case of V1182 Aql (Lorenz et al. 1997, and a paper in preparation). The results are given in Table 2. The third light contribution appears not so large as expected by van Hamme, nevertheless it is important. The velocities given by Gulliver et al. were of course strongly affected by the third light, namely $K_{1}$ was found too small. And it is apparent from Fig. 1 of the Gulliver et al. (1985) paper, that $K_{2}$ could be determined only with very low

\footnotetext{
${ }^{1}$ Constant wavelength is used since the heliocentric correction practically did not change during the time interval when the spectra were taken.
}

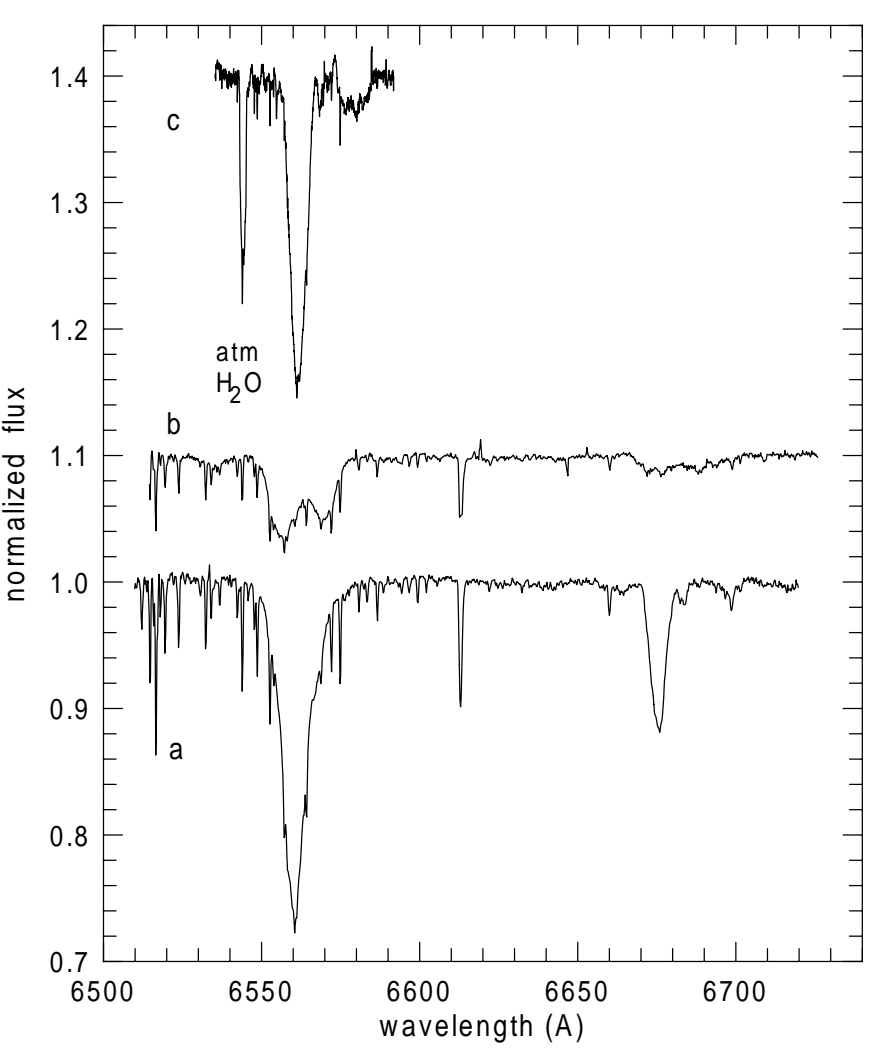

Fig. 7. Profiles of the $\mathrm{H} \alpha$ line in V649 Cas at phase 0.308 a), V382 Cyg at phase 0.285 (b); shifted in flux by +0.10 ), and V431 Pup at phase 0.827 (c); shifted in flux by +0.40 ).

accuracy, since the peak of the cross-correlation function was hardly visible; so our $K_{2}$ differs, too. The newly determined masses are in better agreement with binaries of similar spectral type. The He II 4686 line is quite strong (its equivalent width equals $\approx 0.25 \AA$ ), so the spectral type of the primary component cannot be later than B 0. Though the masses were revised, there is still a discrepancy with too low masses. This problem is known for other binaries as well. In case of V649 Cas it is however necessary to wait for a more reliable spectrum disentangling to confirm this deviation from theory.

The profile of the $\mathrm{H} \alpha$ line is shown in Fig. 7.

The HIPPARCOS satellite measured the brightness of the star on 120 occasions, and the corresponding light curve is presented in Fig. 8 together with measurements by Martin et al. (1990). The scatter in the HIPPARCOS data is considerable, so that a more precise solution of the light curve cannot be expected. For the purpose of obtaining masses of the components, we assumed an inclination of $69^{\circ} .3$ as given by van Hamme for the solution including third light (however note that this solution was obtained for an erroneous mass ratio $q=0.351$ ).

Results of our line fitting are given in Table 1 . When the primary and secondary velocities are solved independently, the systemic velocities differ. Giving the secondary data half weight, the mean systemic velocity is $-11.3 \mathrm{kms}^{-1}$, and the respective solution keeping $V \gamma$ fixed at this value differs only slightly from the individual 


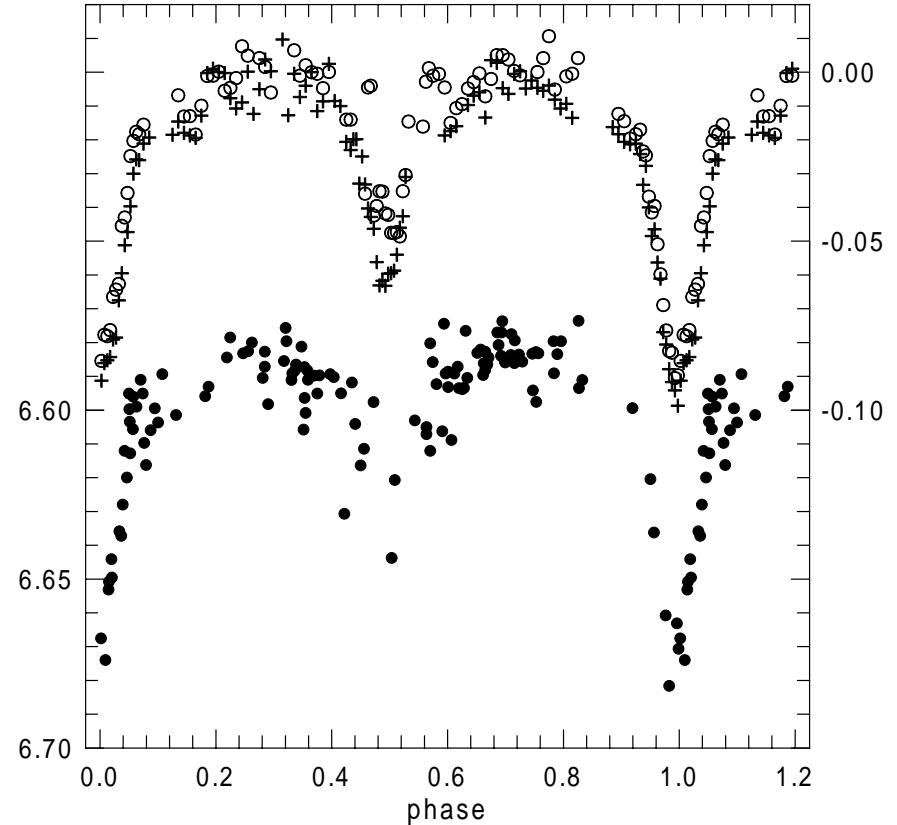

Fig. 8. The light curve of V649 Cas by Martin et al. (1990) in $V$ (crosses) and $B$ (open circles; see the differential magnitude scale at right) and by HIPPARCOS (filled circles, magnitude scale at left).

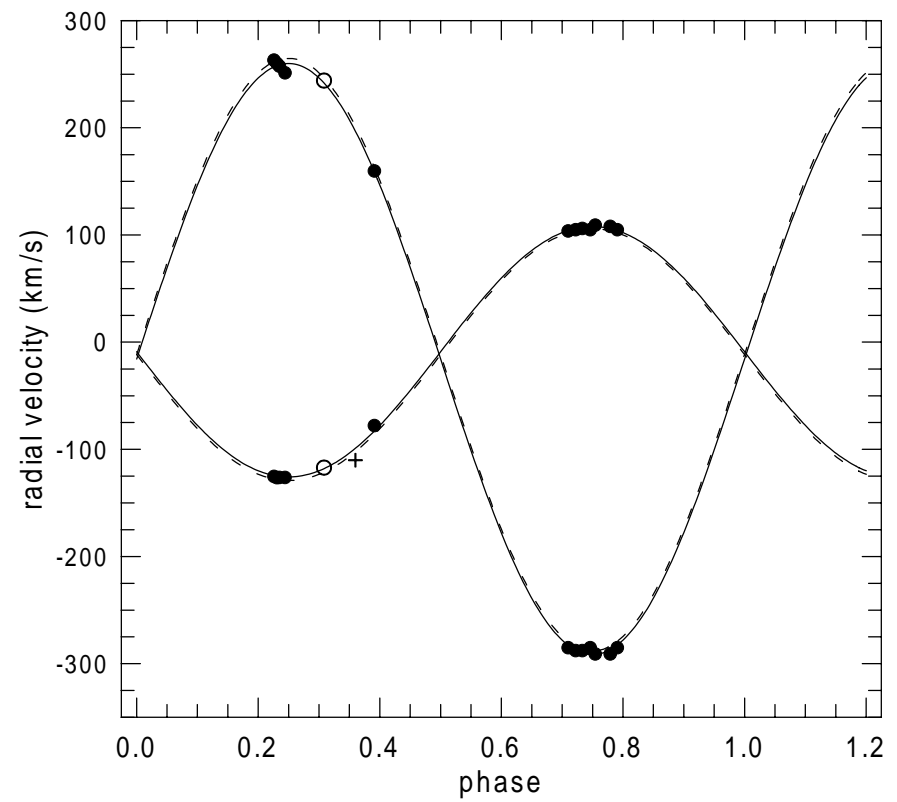

Fig. 9. Radial velocity curve of V649 Cas; filled circles - He I 4922; open circles - H $\alpha$, cross - He II 4686; the dashed line represents the solution with $V_{1} \gamma=V_{2} \gamma$.

solutions with different $V \gamma$ values for both components. A better coverage of the radial velocity curve is needed to disentangle the three spectra more reliably. The curve is shown in Fig. 9. It is encouraging that the $\mathrm{H} \alpha$ and He II 4686 line measurements lie close to the curve (which is mainly defined by the He I 4922 line). We assume that the He II 4686 line is produced only by the primary component, since the secondary as well as the tertiary

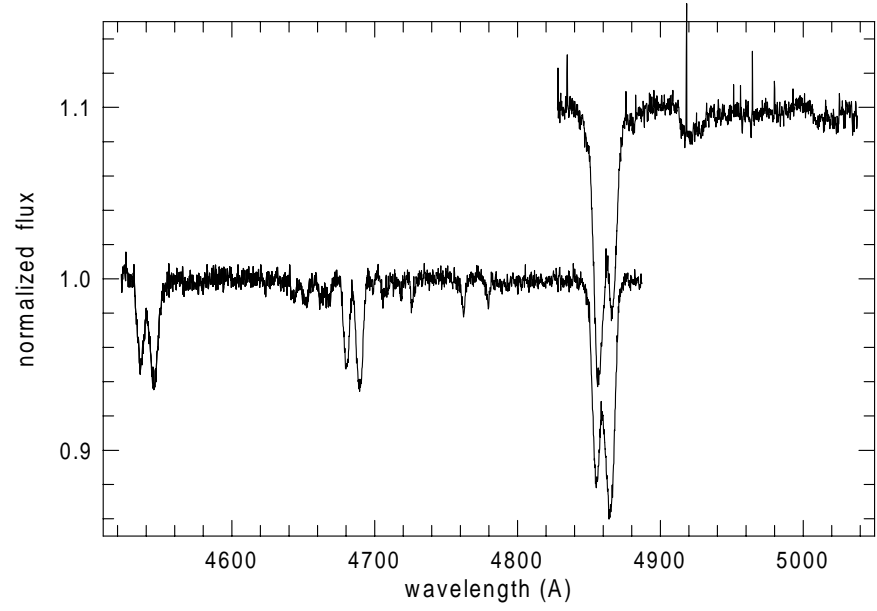

Fig. 10. Spectrum of V382 Cyg; at approximate phase 0.75 for $\lambda 4523$ to $4887 \AA$, at phase 0.29 for $\lambda 4828$ to $5038 \AA$ (shifted in flux by +0.10$)$.

Table 5. Comparison of V382 Cyg parameters.

\begin{tabular}{lccc}
\hline \hline Author & $\begin{array}{c}K_{1} \\
\left(\mathrm{~km} \mathrm{~s}^{-1}\right)\end{array}$ & $\begin{array}{c}K_{2} \\
\left(\mathrm{~km} \mathrm{~s}^{-1}\right)\end{array}$ & $\begin{array}{c}V \gamma \\
\left(\mathrm{km} \mathrm{s}^{-1}\right)\end{array}$ \\
\hline Popper (1978) & 255 & 360 & \\
Popper \& Hill (1971) & $276 \pm 4$ & $400 \pm 6$ & \\
Harries et al. (1997) & $260.2 \pm 2.8$ & $350.8 \pm 3.8$ & $8 \pm 3.6$ \\
Harries et al. (corr.) & 271.1 & 352.0 & 8 \\
\hline
\end{tabular}

components should have lower temperature. The velocity of the third line is $-35 \mathrm{kms}^{-1}$, a value clearly different from the systemic velocity. If the system is considered as gravitationally bound, then this difference should change with time. Since the difference is large, the change should be observable within a few years. It would be certainly very interesting to confirm and monitor such radial velocity changes caused by a suspected third body.

\section{V382 Cyg}

Orbital elements of V382 Cyg (HD 228854) were published by Pearce (1952), but without the measured radial velocities. Popper (1978) obtained spectra of various dispersions and measured velocity amplitudes for several lines. Later Popper's spectra with a reciprocal dispersion of $45 \AA \mathrm{mm}^{-1}$ were analyzed by Popper \& Hill (1991) using a cross-correlation method. In a recent paper by Harries et al. (1997) the radial velocity curve was obtained using 17 CCD spectra in the wavelength region 4700-5950 , with a mean reciprocal dispersion of $0.3 \AA$ pixel $^{-1}$. Results of these studies are listed in Table 5.

Light curves for this binary were measured several times, always using standard $U B V$ filters (Landolt 1964, 1975; Bloomer et al. 1979; Değirmenci et al. 1999). The light curve analysis shows that the binary is in a contact configuration, with an orbital inclination of about $85^{\circ}$. The period is variable (e.g., Mayer et al. 1998; Değirmenci et al. 1999), probably due to strong mass loss via stellar 


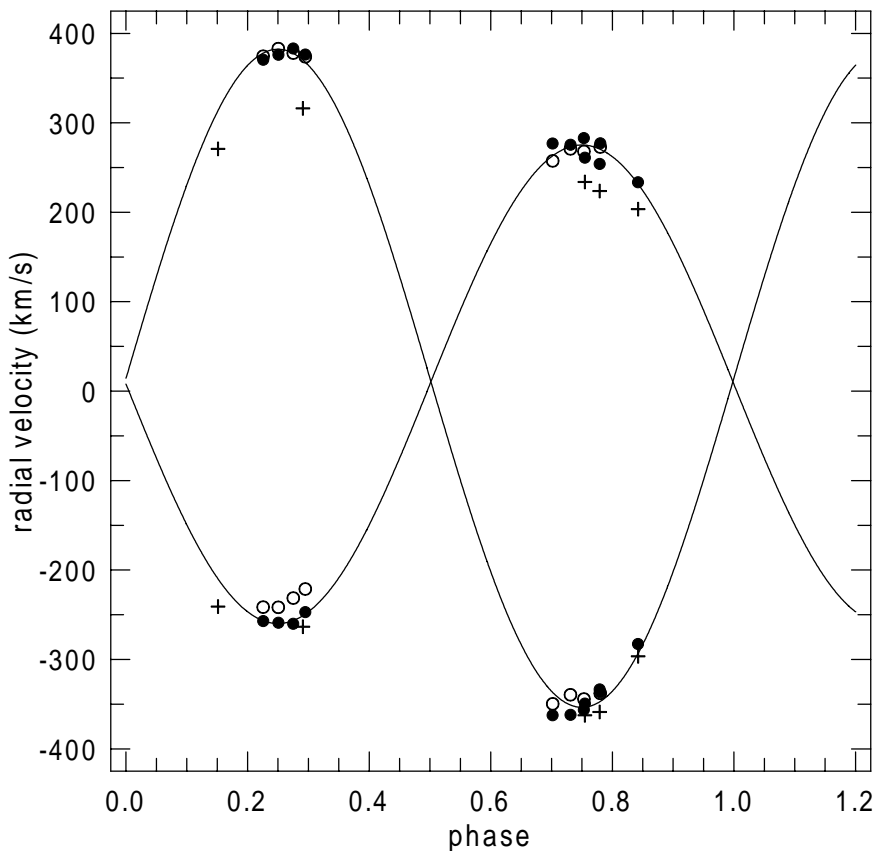

Fig. 11. Radial velocity curve of V382 Cyg; open circles - He II 4542, filled circles - HeII 4686, crosses - H $\beta$.

wind (Koch et al. 1979). Note that the variability of the period was not taken into account by Harries et al. (1997). The correct ephemeris for the epoch of their spectra was

Prim. min. $=$ HJD $2436814.680+1$ d $8855328 \cdot E$

(this ephemeris is valid for our spectra, too). The phases of the Harries et al. velocities should then be smaller by 0 p 025 , and recalculated $K_{1}$ and $K_{2}$ are larger by several kilometers, see Table 5.

Examples of our spectra are displayed in Fig. 10, and for $\mathrm{H} \alpha$ in Fig. 7. To find the radial velocity curve, we gave highest weight to the He II 4686 line, since both its components are narrower than in other lines, and hence can be better resolved. Our results are listed in Table 1, and the radial velocity curve is presented in Fig. 11.

As judged by the weakness of the He I lines 4713 and 4922 , the star is considerably earlier than O 8, i.e. the classification by Pearce (1952) as O 6.5 for the primary and O 7.5 for the secondary appears more correct than that by Hiltner (1956) (O 8). It should be remarked that the equivalent widths, as well as FWHMs, are larger for He II 4541 than for He II 4686 line. According to atmospheric models (Napiwotzki 2001) the equivalent widths of He II 4686 should be larger than that of He II 4541; but the models do explain the larger $F W H M$ of He II 4541. One may compare the V382 Cyg spectra with those of other O-type stars published by Walborn \& Fitzpatrick (1990); among supergiants, He II 4686 appears as an emission line. In our spectra of V382 Cyg, He II 4686 is a net absorption line, though an emission contribution probably reduces the absorption strength. One effect will be that in near quadrature spectra the emission will be most evident at wavelengths between the two binary components, i.e.

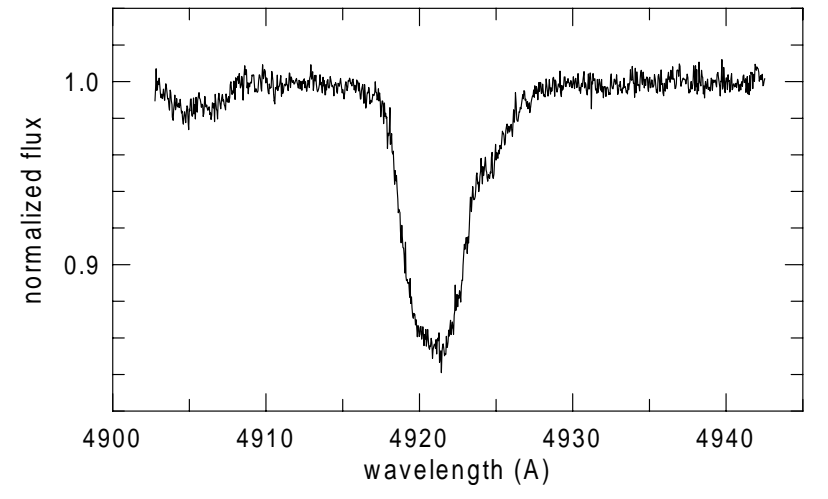

Fig. 12. The He I 4922 line of V431 Pup at phase 0.686, showing features of both binary components.

Table 6. Radial velocities of V431 Pup published by Feast et al. (1955).

\begin{tabular}{cccc}
\hline \hline $\begin{array}{c}\mathrm{JD} \\
-2400000\end{array}$ & $\begin{array}{c}\text { Velocity } \\
\left(\mathrm{km} \mathrm{s}^{-1}\right)\end{array}$ & $\mathrm{Ph}(\mathrm{HIP})$ & $\mathrm{Ph}($ new $)$ \\
\hline 34373.447 & -115 & 0.391 & 0.770 \\
34387.432 & 94 & 0.885 & 0.263 \\
34392.472 & -85 & 0.423 & 0.802 \\
34396.363 & 135 & 0.839 & 0.217 \\
34425.298 & 114 & 0.929 & 0.306 \\
34428.349 & -7 & 0.255 & 0.632 \\
\hline
\end{tabular}

around $V_{\gamma}$, the result would be as observed, and amplitudes of both components should be smaller than derived from the 4686 line. Velocities obtained from He II 4541 would then be more realistic, i.e., both $K_{1,2}$ would be smaller by several percent, and masses would be smaller by about $10 \%$.

Another effect of an emission contribution would be that the weakness of the 4686 line relative to HeII 4541 as observed in our spectra would be in better agreement with the theoretical line ratio deduced from the models of Napiwotzki (2001).

\section{V431 Pup}

The star HD 69882 was discovered as an eclipsing variable with a period of 9.3634 by the HIPPARCOS satellite (ESA 1997). Our spectra were taken before the binary character of the star was known, and, of course, without knowledge of its ephemeris, so the phase coverage is not very good. An example of the He I 4922 region is plotted in Fig. $12, \mathrm{H} \alpha$ in Fig. 7. The secondary line is only discernable - at favourable phases - as an extended wing of the 4922 primary line, and hence its position is only poorly determined. The profile shown in Fig. 12 was obtained from a CAT/CES spectrum. The ECHELEC spectra are noisier, and the secondary line positions are uncertain.

Feast et al. (1955) published radial velocities obtained in six nights. These velocities are listed in Table 6 ; the values are means formed from measurements made by various observers for a given plate. Secondary lines could 


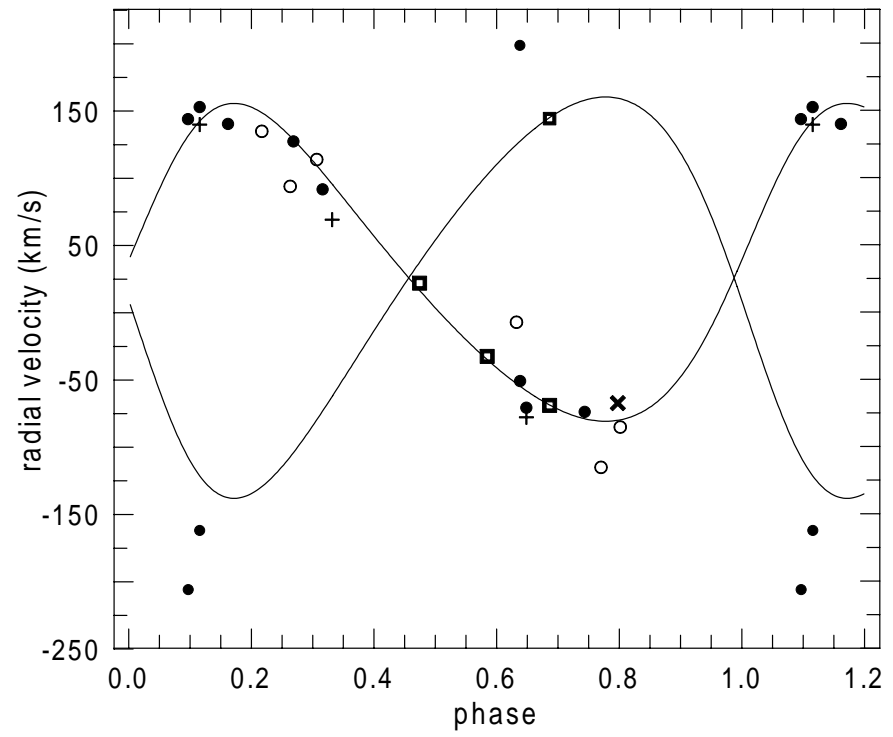

Fig. 13. Radial velocity curve of V431 Pup; filled circles ECHELEC data for HeI, squares - CAT/CES He I data, x - CAT/CES H $\alpha$, crosses - H $\beta$ ECHELEC data, open circles - values by Feast et al. (1955); the curves correspond to the parameters listed in Table 7.

Table 7. Parameters of V431 Pup.

\begin{tabular}{cc}
\hline \hline Parameter & Value \\
\hline$i$ & $68.2^{\circ}$ \\
$K_{1}$ & $118.2 \pm 0.6 \mathrm{~km} \mathrm{~s}^{-1}$ \\
$K_{2}$ & $149.2 \pm 0.6 \mathrm{~km} \mathrm{~s}^{-1}$ \\
$V_{1} \gamma$ & $25.9 \pm 0.7 \mathrm{~km} \mathrm{~s}^{-1}$ \\
$e$ & 0.193 \\
$\omega$ & 300.5 \\
$T_{\text {periastron }}$ & JD 2448509.038 \\
Prim. min. & JD 2448512.553 \\
$r_{1}$ & 0.324 \\
$r_{2}$ & $0.125^{a}$ \\
$a \sin i$ & $48.5 R_{\odot}$ \\
$m_{1} \sin ^{3} i$ & $9.16 M_{\odot}$ \\
$m_{2} \sin ^{3} i$ & $7.50 M_{\odot}$ \\
\hline
\end{tabular}

${ }^{a}$ Assumed.

not be recognized on these low-dispersion plates. In the column labeled " $\mathrm{Ph}(\mathrm{HIP})$ " phases calculated according to the ephemeris by HIPPARCOS are given. However, such phases are incompatible with velocities measured in our spectra. We found that a phase shift of about +0.38 is needed to bring both sets in agreement. Such phase shift means that the true period should be longer than the HIPPARCOS value. Since the difference of epochs between the Feast et al. data and our data is approximately 1570 , the period has to be longer by about 0.0024 . However note that this corresponds only to the smallest possible phase shift; the phase shift might also be -0.62 , or by one or more epochs larger, so that the true period could differ by more.

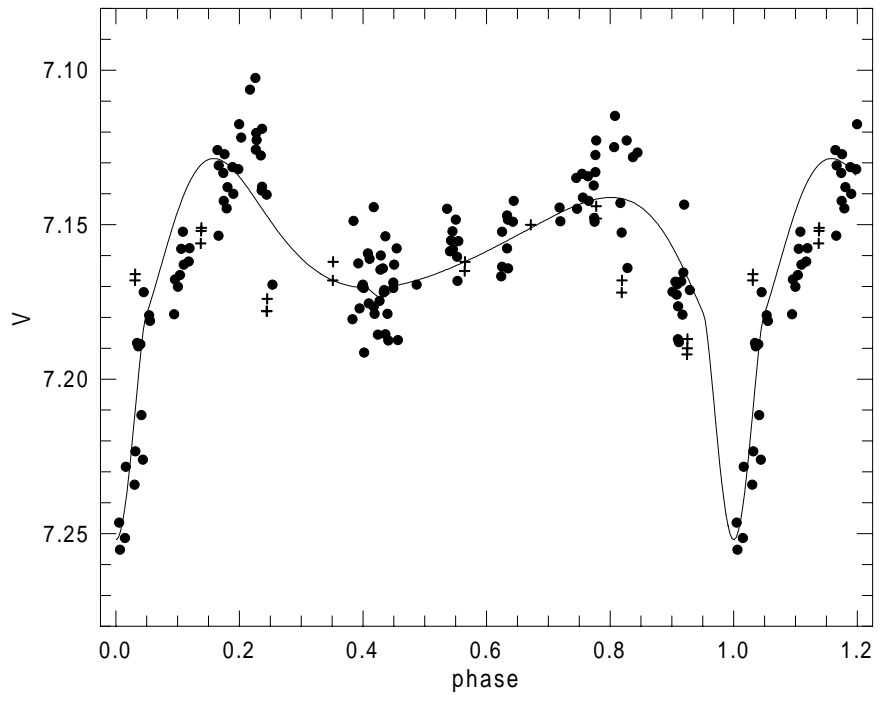

Fig. 14. Our $V$ measurements (crosses) and HIPPARCOS photometry (filled circles) of V431 Pup; the curve is the result of FOTEL.

In April 1994, we obtained several $U B V$ measurements of the star. The color indices were $B-V=$ 0.316 and $U-B=-0.605$. The $V$ magnitudes, together with the HIPPARCOS data, are plotted in Fig. 14. The HIPPARCOS data were transformed to $V$ magnitudes using the formula by Harmanec (1998); $0{ }^{\mathrm{m}} 079$ was subtracted.

To solve the light curve as well as the radial velocity curve, we applied the code FOTEL (Hadrava 1990, 1995), which solves the light and velocity curves simultaneously. The radial velocity curve is plotted in Fig. 13.

In our spectra, radial velocities from lines He I 4922, $\mathrm{H} \beta$ (see below) and $\mathrm{H} \alpha$ can be measured fairly well. However, in spectra where only the shorter wavelengths are covered and $\mathrm{H} \beta$ is affected by a CCD defect (the first three spectra are concerned) the lines present are mostly blends, or are rather weak. The best line here is 4649 , the blend of several CiII and O II lines. Not knowing in advance the representative laboratory wavelength of this blend, we measured this line and by comparison with He I lines 4713 and 4922 and $\mathrm{H} \beta$ obtained a central wavelength of this feature of 4649.66. Due to the uncertainty of this value we however did not use the corresponding velocities in our solution.

Radial velocities as well as photometry do not provide sufficient constraints to define the system. It is clear that the deeper minimum is the secondary minimum, in the sense that the smaller, less luminous (and probably also less massive) star with nearly invisible spectral lines is eclipsed. At the phase when the more luminous star is behind the secondary component, the mutual distance of both components is so large that practically no eclipse occurs.

The minima are not well covered by photometry, and the ratio of radii is nearly impossible to obtain; but the ratio of luminosities of both components might be estimated 
Table 8. Absolute parameters of four binaries.

\begin{tabular}{ccccc}
\hline \hline Parameter & $\begin{array}{c}\text { V337 } \\
\text { Aql }\end{array}$ & $\begin{array}{c}\text { V649 } \\
\text { Cas }\end{array}$ & $\begin{array}{c}\mathrm{V}^{2} 32^{a} \\
\text { Cyg }\end{array}$ & $\begin{array}{c}\text { V431 } \\
\text { Pup }\end{array}$ \\
\hline$M_{1}\left(M_{\odot}\right)$ & 17.2 & 12.9 & 29.2 & 11.4 \\
$R_{1}\left(R_{\odot}\right)$ & 9.4 & 6.1 & 10.1 & 16.9 \\
$M_{2}\left(M_{\odot}\right)$ & 6.8 & 5.5 & 21.2 & 9.4 \\
$R_{2}\left(R_{\odot}\right)$ & 7.2 & 4.4 & 8.4 & 6.5 \\
\hline
\end{tabular}

${ }^{a}$ Inclination, $r_{1}$ and $r_{2}$ according to Değirmenci et al. (1999).

using the CAT/CES spectrum where the secondary line is visible as a deformation of the line wing (see Fig. 12). EWs are 1.249 and $0.147 \AA$, i.e. their ratio is 8.5. Assuming that the $E W$ s represent the luminosities of components, the solution given in Table 7 was obtained. With the assumed value of $r_{2}=0.125$ the temperatures do not differ much, and the assumed ratio of luminosities seems appropriate.

In the solution in Table 7 only the secondary line measured in the CAT/CES spectrum was considered. Taking into account also the features visible in the ECHELEC spectra would increase $K_{2}$ to a considerably larger value with a corresponding primary mass of about $20 M_{\odot}$.

The minimum time of the deeper minimum derived from the FOTEL solution comes out very close to the time determined by HIPPARCOS data. The following ephemeris results:

Sec. min. = HJD $2448508.517+9.36575 \cdot E$.

This ephemeris has been used through this paper, since the time of the deeper minimum is well defined and independent of any orbital solution.

Of course the mass ratio is poorly known, being only based on the mentioned deformation of the He I 4922 line. Nevertheless the basic parameters of the system appear acceptable: a somewhat evolved more massive star accompanied by a main sequence component, both of similar temperatures. From the width of the He I line 4922 we find $v \sin i=180 \mathrm{~km} \mathrm{~s}^{-1}$ (primary component). This is considerably more than what would correspond to synchronous rotation; according to Hut (1981) the pseudosynchronous velocity is $116 \mathrm{kms}^{-1}$.

\section{Discussion}

The absolute parameters of our program stars are listed in Table 8. In the case of V337 Aql the radius of the Roche lobe-filling secondary component is considerably larger than the corresponding MS value; this is however common among early-type semi-detached systems. In the case of V649 Cas, the masses and radii of the primary and secondary components resemble main sequence values for types B 0.5 and B 4, respectively (see, e.g., Harmanec 1988). V382 Cyg is probably the earliest known contact system, so a comparison with other objects is difficult. The last case - V431 Pup - belongs to the rare early-type systems with evolved primaries. It might be compared with
V380 Cyg, see the recent study by Guinan et al. (2000), and with V373 Cas (Hill \& Fisher 1987), all three having somewhat longer periods and non-zero eccentricities (similarly to V373 Cas, the primary component of V431 Pup is rotating faster than synchronously). Hopefully, a good phase coverage of the radial velocity curve will allow for an application of a disentangling method in order to determine the radial velocity curves more accurately, especially the one of the secondary component. Of course, better phase and broader spectral coverage would help to derive more definite parameters also in the other cases.

Acknowledgements. We are grateful to Dr. Napiwotzki for providing us synthetic spectra, which could be compared with the observed spectrum of V382 Cyg. We are indebted to colleagues from Hvar Observatory, who measured for us the star V649 Cas (Dr. Bozic, Mr. Ruzdjak, and Mr. Sudar). We also thank the referee Dr. N. Morrell for valuable suggestions.

\section{References}

Alduseva, V. Y. 1977, PZ, 20, 375

Bloomer, R. H., Burke, E. W., \& Millis, R. L. 1979, BAAS, 91, 439

Catalano, F. A., Catalano, S., \& Rodonò, M. 1971, Ap\&SS, 11,232

Cowley, A. P. 1972, AJ, 77, 750

Değirmenci, Ö. L., Sezer, C., Demircan, O., et al. 1999, A\&AS, 134,327

Drechsel, H., Haas, S., Lorenz, R., \& Gayler, S. 1995, A\&A, 294,273

ESA 1997, The Hipparcos and Tycho Catalogues, ESA SP1200, Noordwijk, ESA

Feast, M. W., Thackeray, A. D., \& Wesselink, A. J. 1955, MmRAS, 67, 1

Feast, M. W., \& Thackeray, A. D. 1963, MmRAS, 68, 173

Guinan, E. F., Ribas, I., Fitzpatrick, E. L., et al. 2000, ApJ, 544,409

Gulliver, A. F., Hube, D. P., \& Lowe, A. 1982, IBVS, 2146

Gulliver, A. F., Hube, D. P., \& Hill, G. 1985, A\&A, 151, 254

Hadrava, P. 1990, Contrib. Astron. Inst. Skalnate Pleso, 20, 23

Hadrava, P. 1995, A\&AS, 114, 393

van Hamme, W. 1992, PASP, 104, 242

Harmanec, P. 1988, BAC, 39, 329

Harmanec, P. 1998, A\&A, 335, 173

Harries, T. J., Hilditch, R. W., \& Hill, G. 1997, MNRAS, 285, 277

Hill, G., \& Fisher, W. A. 1987, A\&A, 171, 123

Hiltner, W. A. 1956, ApJS, 2, 317

Horn, J., Kubát, J., Harmanec, P., et al. 1996, A\&A, 309, 521

Hut, P. 1981, A\&A, 99, 126

Jerzykiewicz, M. 1993, A\&AS, 97, 421

Jerzykiewicz, M., \& Sterken, C. 1982, Be Stars, ed. M. Jaschek, \& H.-G. Groth (D. Reidel, Dordrecht), IAU Symp., 98, 49

Koch, R. H., Siah, M. J., \& Fanelli, M. N. 1979, PASP, 91, 47

Kwee, K. K., \& van Woerden, H. 1956, Bull. Astron. Inst. Netherlands, 12, 327

Landolt, A. U. 1964, ApJ, 140, 1494

Landolt, A. U. 1975, PASP, 87, 409

Lorenz, R., Mayer, P., \& Drechsel, H. 1994, A\&A, 291, 185

Lorenz, R., Drechsel, H., \& Mayer, P. 1997, AG Abst. Ser., 13, 208 
Lorenz, R., Mayer, P., \& Drechsel, H. 1998, A\&A, 332, 909

Martin, B. E., Hube, D. P., \& Lyder, D. A. 1990, PASP, 102, 1375

Mayer, P., Lorenz, R., Chochol, D., \& Irsmambetova, T. R. 1994, A\&A, 288, L13

Mayer, P., Lorenz, R., \& Drechsel, H. 1997, A\&A, 320, 107

Mayer, P., Niarchos, P. G., Lorenz, R., Wolf, M., \& Christie, G. 1998, A\&AS, 130, 311

Napiwotzki, R. 2001, private communication

Neubauer, F. J. 1943, ApJ, 97, 300
Pearce, J. A. 1952, PASP, 64, 1952

Popper, D. M. 1978, ApJ, 220, L11

Popper, D. M. 1982, ApJ, 262, 641

Popper, D. M., \& Hill, G. 1991, AJ, 101, 600

Škoda, P. 1996, in Astronomical Data Analysis Software and Systems V, ed. G. H. Jacoby, \& J. Barnes (ASP, San Francisco), ASP Conf. Ser., 101, 187

Wade, R. A., \& Rucinski, S. M. 1985, A\&AS, 60, 471

Walborn, N. R., \& Fitzpatrick, E. L. 1990, PASP, 91, 379

Young, R. K. 1939, Publ. David Dunlap Obs., 1, 71 